\title{
Research Methods in Machine Learning: A Content Analysis
}

\author{
Jackson Kamiri \\ School of Computing and Information Technology \\ Murang'a University of Technology \\ Murang'a, Kenya \\ Email: jkamiri [AT] mut.ac.ke
}

\author{
Geoffrey Mariga \\ School of Computing and Information Technology \\ Murang'a University of Technology \\ Murang'a, Kenya \\ Email: gmariga [AT] mut.ac.ke
}

\begin{abstract}
Research methods in machine learning play a pivotal role since the accuracy and reliability of the results are influenced by the research methods used. The main aims of this paper were to explore current research methods in machine learning, emerging themes, and the implications of those themes in machine learning research. To achieve this the researchers analyzed a total of 100 articles published since 2019 in IEEE journals. This study revealed that Machine learning uses quantitative research methods with experimental research design being the de facto research approach. The study also revealed that researchers nowadays use more than one algorithm to address a problem. Optimal feature selection has also emerged to be a key thing that researchers are using to optimize the performance of Machine learning algorithms. Confusion matrix and its derivatives are still the main ways used to evaluate the performance of algorithms, although researchers are now also considering the processing time taken by an algorithm to execute. Python programming languages together with its libraries are the most used tools in creating, training, and testing models. The most used algorithms in addressing both classification and prediction problems are; Naïve Bayes, Support Vector Machine, Random Forest, Artificial Neural Networks, and Decision Tree. The recurring themes identified in this study are likely to open new frontiers in Machine learning research.
\end{abstract}

Keywords: Research methods in machine learning, machine learning algorithms, machine learning techniques.

\section{INTRODUCTION}

Machine learning can be defined as a field of artificial intelligence that is concerned with the development of algorithms and techniques that allow a computer to learn and gain intelligence from experience [1]. Research methods in machine learning research play a pivotal role since the accuracy and reliability of the results are influenced by the research approach/ method used. In machine learning, models learn from historical data which can either be primary data or secondary data. This creates a wide pool of knowledge from which machines can learn and make decisions based on what they learn [2].

The research articles analyzed emphasized the need to choose an appropriate methodology. Machine learning is classified as scientific research, therefore, it's research methodology according to many scholars incorporates several factors which include; choice of training data to use, choice of data attributes to use, choice of algorithm $(\mathrm{s})$ to use in the model, the ratio between training data and testing data, the performance measures of the algorithms, among other factors. Thus, scholars have demonstrated that the choice of methodology to use should span across the above factors. The objectives of the research also influence the methodology to be used [3]

Scholars in this field of machine learning have demonstrated that a good research methodology should be capable of clearly explaining how the researcher ended-up with the results [4]. Therefore, the methodology should be put in such a way that another researcher can easily follow and end up with the same results. Some researchers have opted to use a single approach in terms of choice of the algorithm while others use more than one algorithm to model and then they compare the results of the various algorithms [4]

Research in the field of machine learning is mainly quantitative since it involves the modeling of data and also using statistical approaches and formulations to make sense of the data [1], [2], [3], [4]. Therefore, the methods used in machine learning research in most cases must be consistent with quantitative research. However, this is not to mean that all fields of artificial intelligence or domains affiliated to machine learning are confiscated to quantitative research. Some areas such as Natural Language Processing may require a qualitative research approach [5].

When scholars are researching on machine learning they must first scrutinize the problem that they intend to solve and then decide if it requires a quantitative approach, qualitative approach, or a hybrid of the two approaches. This is so because machine learning does not exist in a vacuum, it is used to solve problems in society. Thus, the nature of the problem influences the methodology to be used in research [6]. Also, machine learning is a very active area of research, therefore, scholars are continuously trying to solve a majority 
of the problems in the various domains using machine learning techniques.

The articles assessed in this paper have shown that research methodology in machine learning can be the key difference between various study which are basically solving a similar problem in the same domain [7,8]. Therefore, the methodology can form the research gap that a researcher wants to address. The researcher will then use a different approach which s/he thinks is better than the approach $_{(\mathrm{s})}$ used by other researchers previously to solve the same problem. Some researchers have also combined methodologies used by other researchers with the sole aim of increasing the accuracy of the results.

\section{Problem statement}

This study aims at exploring research methods in machine learning and determining emerging themes in the field of machine learning research. Specifically, the following three research questions will be addressed.

1) What research methods are commonly used in the field of Machine Learning?

2) What other recurring themes are there in Machine Learning field with regard to research methods?

3) What implications do these recurring themes in research methods have for the Machine Learning discipline?

Scholars in the field of Machine learning have contributed widely to the evolution of research methods. Therefore, cross-examination of the status quo in the field of machine learning will inform scholars about research methods and help them make more informed decisions while selecting the methodology to use in their research.

\section{LITERATURE REVIEW}

\section{A survey of Content Analysis}

Grossman, Zak, and Zelinsk conducted a quantitative content analysis on mobile applications used by caregivers. In the analysis, 44 applications that self-identified themselves or were advertised as caregivers aiding tool were assessed. The apps were identified in two major application stores namely iTunes App Store and Google play store. To get the apps, the researchers searched the keywords "caregivers", "caregiving" and "elderly care" [9]. The researchers only include applications that exclusively dealt with informal or family caregivers for elderly people. Therefore, apps developed for professional caregivers, and those developed for people with special chronic conditions such as diabetes were excluded [9].
The apps were assessed against five functions that are performed by successful caregivers to determine the level to which the apps relieve the burden of caregivers. The five key functions were; (i) Information and Resources, (ii) practical problem solving involving behavioral solutions, medication management, safety, and personal health records tracking. (ii) Memory aids, (iv) Family communication which includes notification for checkups, emergency contact list, sharing of critical information. (v) caregiver support that is caring for the caregiver. The general assessment of the apps revealed that $50 \%$ (22 apps) were designed for caregivers taking care of people with memory loss [9].

The results of the analysis against the five functions were as follows; first, only 34\% (15 apps) satisfied the criteria of providing caregivers with relevant information and resources such as searchable databases [9]. Second, 21 applications met the criteria for practical problem-solving. Practical problemsolving in this case was defined as addressing medication management, safety health record tracking, and behavioral solutions [9]. Third, 15 apps passed the test of facilitating better communication and enhancing coordination among family members [9]. Fourth, 12 applications met the criteria of actin as a memory aid. The apps have tools that enable people with dementia to enhance their cognitive abilities [9]. Five, a total of 10 apps of the 44 apps contained tools that care for the caregiver by providing emotional support stress management, and social support, among others to the caregivers [9].

[10] conducted a content analysis of research methods in Library Information Science (LIS). The researcher analyzed a total of 1162 articles published between 2001 and 2010 in three major journals of LIS namely; Journal of Documentation (JDoc), Journal of the American Society for Information Science, and Technology (JASIS\&T), and Library Information Science research (LIS). However, the researcher deferred the analysis of articles in the JASIS\&T journal between 2003-2008 and only analyzed articles between 2001-2002 and 2009-2010 [10]. The articles were analyzed quantitively and qualitatively to address some recurring themes about research method selection and application in the scholarly domain. Non-research articles in this field were excluded from the analysis.

The findings of the research were as follows: the three journals shared 4 of the top five research methods identified in this study. The theoretical approach leads with a cumulative percentage of $65 \%$, the content analysis took the second position with a percentage of $57 \%$, Questionnaire becomes position three with a cumulative percentage of $55.8 \%$, and experiment occupied position four with a $53.4 \%$ cumulative percentage score [10]. The researcher discovered that unlike before, questionnaires and surveys no longer dominate as the leading research methods in Library Information Science [10]. Despite Bibliometrics leading in JASIS\&T, it did not feature well in the other journals, 
therefore, it did not qualify to be among the top 4 techniques. The researcher further reports that the use of multiple methods has gained a lot of prevalence.

Azeez and Van der Vyver conducted a comprehensive content analysis on privacy and security issues in e-health cloud-based model. The scholar reviewed 110 articles in this area and discovered several models discovered in their solutions. The articles were sourced from the following journals; ACM digital library, IEEE digital library, IEE explore digital library, Springer, Elsevier, and Science direct [11]. The 110 reviewed articles were arrived at after comparing the models and approaches used by many researchers. The researchers examined the strengths and weaknesses of each method adopted in addressing E-health security challenges [11].

The scholars discovered that for any E-health system to be reliable it must have strong mechanisms to counter various security threats such as; tampering, masquerading, denial of service attack, and privacy [11]. Public Key Encryption was identified as the most widely used security mechanism, however, the researcher record that it needs to be enhanced. $\mathrm{ABE}$ is another security scheme that is being used. $\mathrm{ABE}$ is efficient in enhancing security and privacy although it is expensive considering computational complexity. The high cost of $\mathrm{ABE}$ is mainly because the ciphertext is expensive thus decryption is expensive [11].

Selective encryption was also widely used in the articles assed. This involves encrypting the parts of the data that are considered to be more sensitive [11]. This method is considered to be less costly, therefore, researchers are considering it over ABE. The researchers concluded that research in security and privacy issues in E-health is still wanting since threats are increasing with time.

\section{METHOD}

One hundred research articles published since 2019 were obtained from IEEE journals. All the research papers used were in the field of machine learning. Journal papers that were not written in English or at least translated to English were excluded. Access to these journals was provided by Murang'a University of Technology.

The data collection yielded 100 research articles in the field of machine learning as summarized on table1. These articles were considered to be very relevant since they were not more than 2 years old, thus they can inform current research in the field of machine learning which is a very active field. The research articles chosen were solving different problems using machine learning approaches. All the 100 articles were analyzed by the researchers to determine the research methods used in machine learning.

In this study, the researchers analyzed each of the sampled papers along several dimensions to describe machine learning research methods. These dimensions are: General research design, sources of data, algorithms used, and approaches applied in data pre-processing, model training, testing, and evaluation. The above issues were used to inform the findings of this paper. All the 100 research papers used in this study had the

above key parameters in their research methodology. These parameters are just general parameters, therefore, under each one of them, several sub-parameters were identified. For example, under the performance metrics, the majority of the articles recorded the performance of the algorithms used under the various performance metrics.

TABLE 1: RESEARCH ARTICLES ANALYZED

\begin{tabular}{|c|c|c|c|}
\hline ID & Application Area & Number of Articles & References of Analyzed Articles \\
\hline 1 & $\begin{array}{l}\text { Machine Learning Algorithm } \\
\text { Enhancement }\end{array}$ & 5 & [16], [85], [88], [89], [95] \\
\hline 2 & Solutions in Engineering & 8 & [14], [27], [62], [76], [86], [92], [110], [114] \\
\hline 3 & $\begin{array}{l}\text { Solutions in computer networks and } \\
\text { security }\end{array}$ & 23 & $\begin{array}{l}{[17],[40],[49],[50],[51],[63],[64],[65],[68],} \\
{[70],[72],[80],[93],[94],[99],} \\
{[101],[103],[104],[105],[106],[113],[120],[122],} \\
{[123]}\end{array}$ \\
\hline 4 & Natural Language Processing & 8 & {$[28],[20],[44],[47],[74],[96],[98],[112]$} \\
\hline 5 & Image Processing & 10 & $\begin{array}{l}{[29],[32],[37],[55],[69],[73],[77],[84],[87],} \\
{[119]}\end{array}$ \\
\hline 6 & Solutions Health & 24 & $\begin{array}{l}\text { [1], [30], [31], [19], [34], [23], [43], [46], [48], [53], } \\
\text { [57], [61], [75], [78], [81], [82], [83], [90], [97], } \\
{[111],[115],[116],[118],[124]}\end{array}$ \\
\hline 7 & Solutions in Software Engineering & 1 & [39] \\
\hline 8 & Solutions in Internet of Things & 5 & {$[41],[54],[91],[100],[121]$} \\
\hline 9 & Solutions in Education & 2 & {$[42,45]$} \\
\hline 10 & $\begin{array}{l}\text { Solutions in Analytics and Artificial } \\
\text { Intelligence }\end{array}$ & 13 & $\begin{array}{l}\text { [52], [3], [58], [59], [60], [66], [67], [79], [102], } \\
{[107],[108],[109],[117]}\end{array}$ \\
\hline
\end{tabular}


Table 1 shows a summary of research articles analyzed in this research. The table portrays that machine learning techniques are being are applied to address problems in diverse fields.

\section{RESULTS}

This section presents results of data analysis along the dimensions indicated in section 1.3 .

\section{General Research Design}

Findings of this study indicates that research in machine learning is mainly quantitative. Therefore, it has standardized approaches and uses statistical models in the various segments of the research. All articles analyzed in this study used an experimental research design. This involved the design of an experiment and conducting the experiment to obtain results. The general approach used in conducting experiments in all the analyzed research articles is as follows: Data collection, Data pre-processing, Model training, model testing, model evaluation. These steps are summarized in Figure 1.

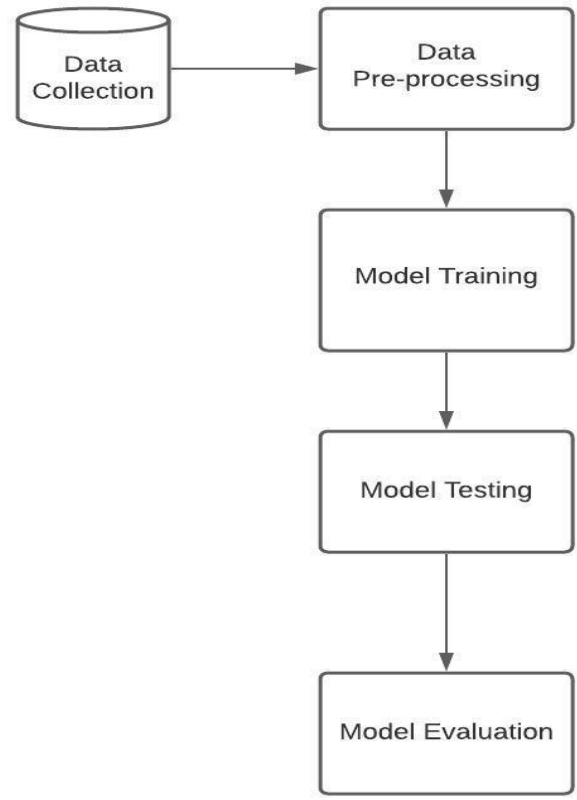

Figure 1: General Research Approach

\section{Data Collection}

In this stage, two main sources of data were used in the articles analyzed. The two sources are secondary data and primary data. $80 \%$ of the articles used purely secondary data sets in the research as shown in Figure 2. The secondary data set was obtained from data repositories. The main used data repository was the UCI ML repository. This repository has been used by research works in machine learning that are addressing diverse problems. This shows that the repository has a variety of datasets in different areas. Apart from repositories, some research articles used data obtained from government entities and Non-governmental organizations.

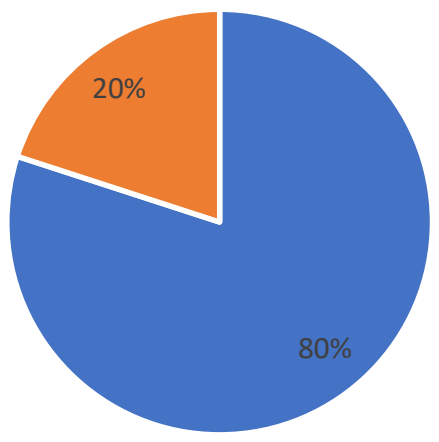

- Secondary data Only = Primary + Secondary Data

Figure 2: Type of Data Used

Also, of the researchers who used secondary data, $87 \%$ used a single data set while $13 \%$ used more than one data sets for example [17] has used three datasets as summarized in Figure 3. Among the researchers who used more than one dataset, the majority sourced some data set from repositories and some of the data set from either governmental or nongovernmental organizations. Others sourced all their data sets from repositories. The key reason for using more than one dataset according to the researchers was to enhance the validity and reliability of the model [17].

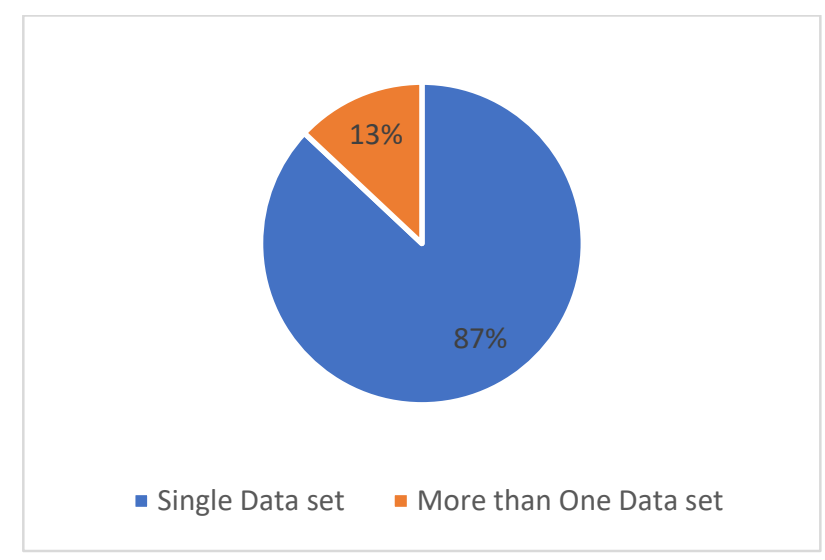

Figure 3: Secondary data usage analysis

Primary data was mainly sourced from recording data over a long period. Research that involved primary data took a longer time than those that used secondary data. A combination of both primary and secondary data was used 
where there is a need to guarantee the validity of the model or to enhance the model to accommodate real-time data [12]. Also, where secondary data is not available or the available secondary data is not sufficient then the researchers resulted in using either purely primary data or a combination of both primary and secondary data.

None of the articles analyzed in this study used more than one set of primary data. Also, none of the research works used qualitative data collection methods to collect any form of primary data. This, therefore, proves that research in machine learning is purely quantitative.

\section{Data Pre-processing}

All the articles analyzed had this stage as part of their methodology. The majority of the articles noted that this is a very critical stage in machine learning since this stage can influence the performance of models and consequently influence the results of the model. This is so because machine learning algorithms learn from the data provided to them and use the acquired knowledge to make decisions.

The analyzed articles revealed that despite the algorithms to be used in the research (supervised, unsupervised, or reinforcement learning) or the nature of the research (classification or regression) data pre-processing is a paramount stage that cannot be omitted. Some articles recorded that much focus in their research work was on data pre-processing.

Data pre-processing involved a series of activities. The activities that were unanimous among all analyzed research articles were; data cleanup, data normalization, and noise reduction e.g. [17], [18]. $10 \%$ of total analyzed articles performed feature extraction/ optimal feature selection in data pre-processing as illustrated in Figure 4. Data cleaning involved activities such as filling-in null values or eliminating null values if they cannot be filled.

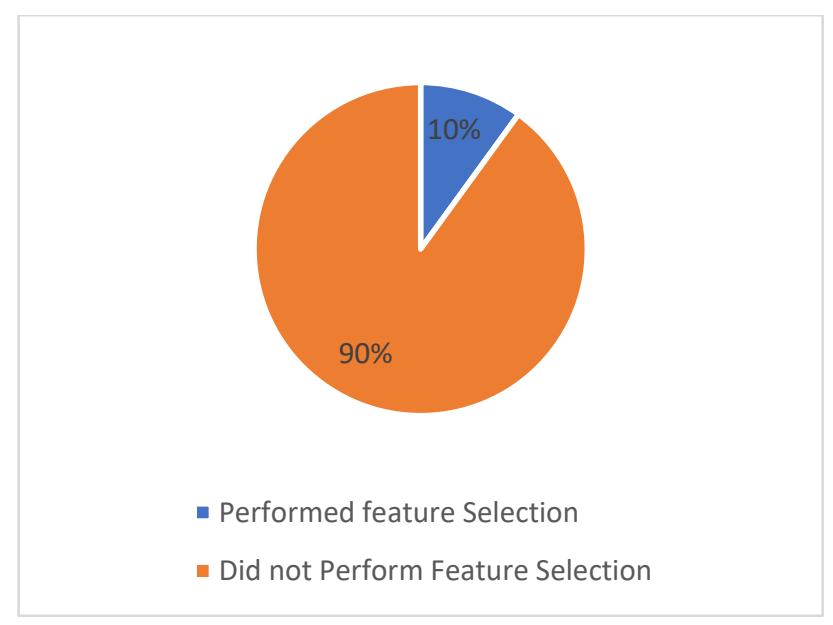

Figure 4: Data Pre-processing
Data normalization Involved tasks such as formatting the data in a form that is easier for the algorithms to understand during training. It also involved data scaling and ensuring that the training data is in the same format as the testing data. It was noted that normalized data promotes the efficiency of machine learning models [17].

Noise reduction involved the elimination of outliers or any data item that is inconsistent with the other parts of the data. Optimal feature selection involved the selection of critical features that influence the results also known as the dependent variable in a great way. Articles that applied this technique first modeled the data without extracting optimal features and then in the second phase the optimal features were selected and the data modeled again.

In all the articles that used optimal feature extraction, it was noted that the performance of the models in terms of accuracy and also in the confusion matrix improved after optimal features were selected [13], [14], [15], [16]. This is a new development in the field of machine learning that is attracting a lot of interest among researchers since it improves performance.

Principle component analysis PCA has been used as one of the major techniques of selecting optimal features [13], [14]. It identifies correlation among various attributes of a dataset, therefore, enabling researchers to determine how each attribute influences the results and the relevance of such attribute in that particular data set [14].

\section{Choice of algorithms}

All the articles analyzed used at least one machine-learning algorithm to solve the research problem that they were addressing. It was noted that the choice of algorithm depends mainly on the nature of the problem to be solved. According to the analyzed articles, problems can be grouped as either classification problems or regression/ prediction problems. Also, problems can be classified as either supervised learning or unsupervised learning problems.

Some Machine learning algorithms can be used to solve classification problems only while others can be used to solve regression problems only, while others solve both classification and regression problems. It was noted that algorithms that solve both classification and regression problems have attracted a lot of favor from the researchers as per the articles analyzed.

The main pure classification algorithm used are K-Nearest Neighbors and Logistic regression. The main purely prediction algorithm used is Linear regression. The most commonly used algorithms that support both classification and prediction are; Naïve Bayes, Support Vector Machine, Random Forest, Artificial Neural Networks, and Decision Tree as illustrated in Figure 5. 


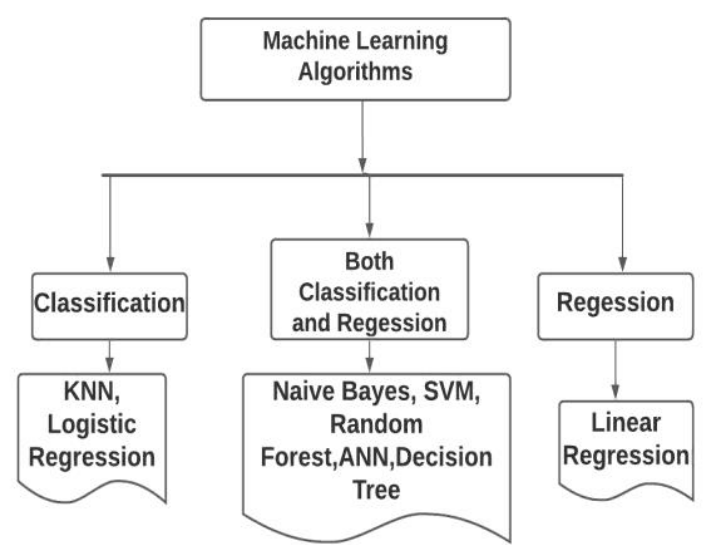

Figure 5: Main Algorithms used

It was noted that the above algorithms which support both classification and prediction have modules within them that perform a specific task. For example, it was identified that Convolutional Neural Networks is part of Artificial Neural Networks that deals with image classification [19]. This enables the algorithms to perform numerous tasks in both classification and regression. Among the analyzed articles, $53 \%$ have specified the specific element of such algorithms that they used in their study. This is a new paradigm shift where researchers are now not just concerned about the algorithm to use in solving a problem but also the specific module of the algorithm that if used will generate optimal results [20].

$98 \%$ of the analyzed articles used more than one algorithm to model as illustrated in Figure 6. Some used as many as five algorithms to model the data and then compared the results of the various algorithms. It was noted that this was aimed at increasing the reliability of results obtained since in using more than one algorithm, the results become immune to the limitations of any particular algorithms.

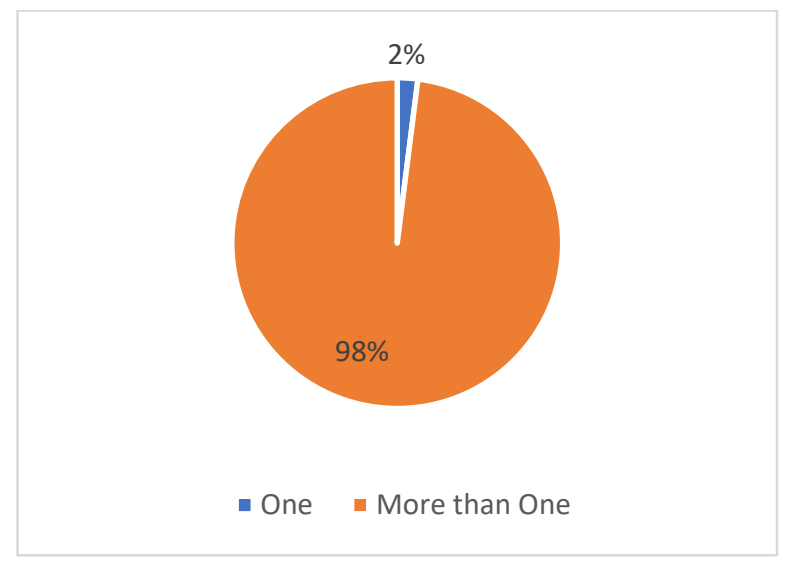

Figure 6: Number of algorithms used
The analyzed articles revealed that some algorithms such as Neural networks algorithms may take more processing time when dealing with large sets of data but will generate better results in terms of accuracy. Others like Decision trees perform better with small sets of data; however, the decision tree can be unstable. Support vector machine can handle complex functions and even scale up with dimensional data, however, it is not suitable with outliers [21].

The above critical features of algorithms influenced their performance and thus the nature of the data used determined which algorithm will register the highest performance. It was discovered that by using multiple algorithms, researchers can discover the most suitable algorithm for a given problem.

\section{Model Training and Testing}

All the analyzed articles included model training as part of their methodology. $80 \%$ of the articles analyzed in this study used secondary data to train the model of these, $83 \%$ used a single data set for both training and testing. The data set was broken down into a training set and a testing set. The majority used $70 \%$ of the data for training while the remaining $30 \%$ was used for testing. Others used $75 \%$ of the data for training and $25 \%$ of the data was reserved for testing.

$20 \%$ of the total articles analyzed used 10-fold crossvalidation to train and test the model. In this approach, the data set was divided into 10 equal parts. 9 parts or segments were used to train the model and then the remaining 1 segment was used to test the model. This was repeated 10 times with the testing segment being alternated among the 10 equal parts. Therefore, each part was used for testing once and for training 9 times.

$13 \%$ of the researchers who used secondary data used different data sets for training and testing. Some used two data sets in their research. One set was used for training while the other set was used for testing the model. Some researchers though very few used secondary data to train the model and primary data to test the model.

It was discovered that researchers who used different sets to train and test their models had to do a lot of data preprocessing to ensure that optimal features in both training and testing data sets are well identified and also the attributes are properly labeled so that the model does not get confused.

\section{Model Evaluation}

The evaluation matrix that was used by all the researchers in the articles analyzed is the confusion matrix. The researchers who used more than one algorithm evaluated the performance of each algorithm in the confusion matrix and its derivatives. The other measure that $40 \%$ of the researchers used on top of the confusion matrix is the processing time taken by each algorithm as shown in Figure 7. 


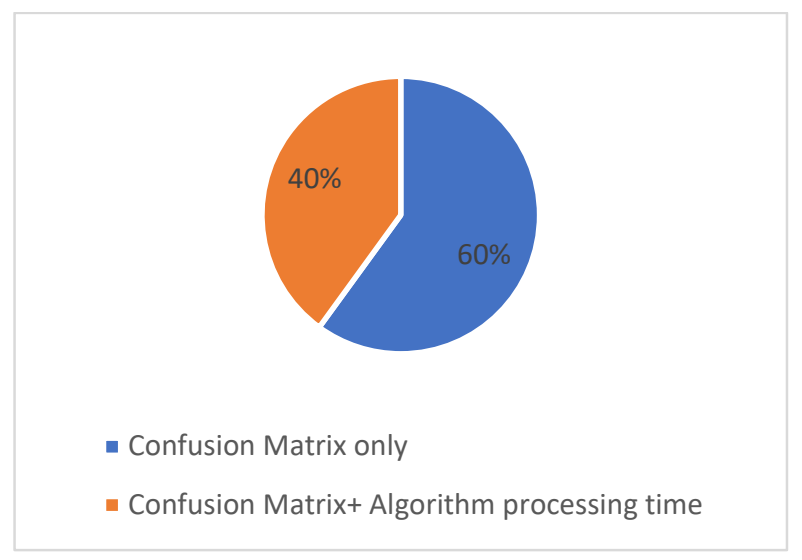

Figure 7: Model Evaluation

The confusion matrix has the following parameters which were used by the researchers to come up with the derivatives of the confusion matrix [22].
a) True Positive (TP)
b) True Negative (TN)
c) False Positive (FP)
d) False Negative (FN)

The derivatives of these matrices are [22,23];
a) Accuracy- $(\mathrm{TP}+\mathrm{TN}) /(\mathrm{TP}+\mathrm{TN}+\mathrm{FP}+\mathrm{FN})$
b) Precision $=(\mathrm{TP}) /(\mathrm{TP}+\mathrm{FP})$
c) Recall $=(\mathrm{TP}) /(\mathrm{TP}+\mathrm{FN})$ $\mathrm{F}-\mathrm{Score}=2 * \quad$ (precision $\quad *$ Recall $) / \quad($ Recall+
Precision $)$

Evaluation of algorithms against the above parameters generated a true picture of how the algorithm has performed. Some researchers were interested in only the accuracy of the algorithm while others were concerned about the performance of algorithms against all the parameters of the matrix.

It was discovered that some researchers considered running the models more than once and recorded the performance of each of the test runs, then computed the average performance. It was also discovered that the researchers who considered processing time as a measure of performance, went a step further and evaluated processing time against the accuracy of each algorithm in their research to determine the most optimal algorithms.

One new approach that was used to evaluate the processing time against the accuracy of each algorithm was the use of regression equations $\mathrm{X}$ and $\mathrm{Y}$ [24].

\section{Tools and Techniques used}

$89 \%$ of the articles analyzed used python programming language to develop the model, train the model, and test the model. The most utilized python library is the Scikit-Learn library. $10 \%$ of the analyzed articles used R to develop, train, and test the model. $1 \%$ used a combination of machine learning approaches implemented using python and Fuzzy logic implemented in MATLAB.

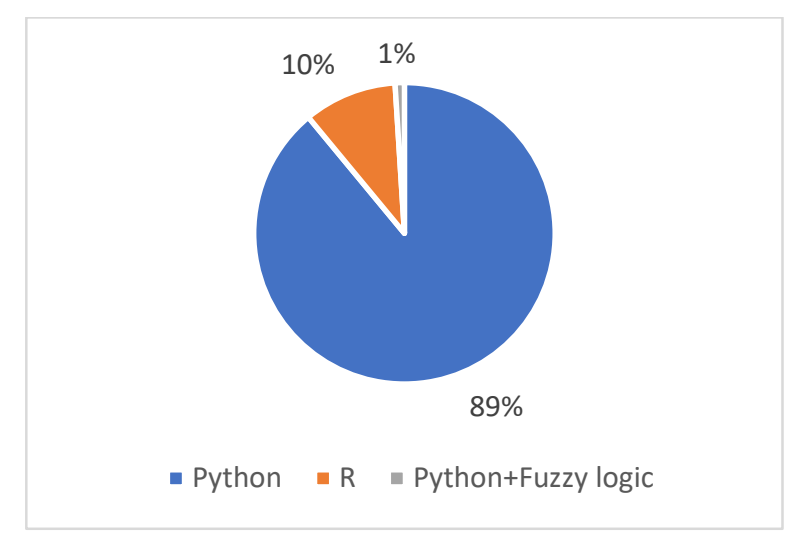

Figure 8:Programming Techniques Used

This shows that Python programming language and its libraries are gaining a lot of preference among researchers in the field of machine learning as opposed to R. Python is delivering an easier and more efficient and effective way of doing machine learning research. Also, the vast libraries in python help researchers to perform numerous activities on the data or models with a lot of conveniences.

\section{DISCUSSION}

This study has discovered that research in Machine learning is attracting a lot of interest among researchers which is a clear demonstration that Machine Learning is a very active area of research.

The most recurring themes in machine learning research include;

i. The use of algorithms that can perform both classification and prediction

ii. The use of more than one algorithm to address a problem to enhance reliability

iii. Optimal feature selection in data pre-processing as a way of improving performance

iv. The use of specific modules of an algorithm to address a problem in more effective and efficient ways.

v. The use of both processing time and confusion matrix derivatives to evaluate machine learning models.

These recurring themes are likely to open new frontiers in machine learning research. Also, due to the active nature of this field of research, more techniques are likely to pop-up with time. 


\section{CONCLUSION AND FUTURE WORK}

The key contribution of this study is that it not only provides an updated view of research methods in the field of Machine Learning but also discusses emerging issues that are worth the attention of researchers in the field of machine learning. The findings of the study should help Machine Learning researchers to make informed decisions while pursing research in machine learning.

This study has focused more on general research methods in machine learning. Future researchers can perform a content analysis of specific areas of machine learning such as supervised learning, unsupervised learning, video analytics, text analytics, classification, and prediction.

\section{REFERENCES}

[1] H. Kaur and V. Kumari, "Predictive modelling and analytics for diabetes using a machine learning approach", 2020 Applied computing and informatics.

[2] X. Li, K. Li, D. Qiao, Y. Ding and D. Wei, "Application Research of Machine Learning Method Based on Distributed Cluster in Information Retrieval," 2019 International Conference on Communications, Information System and Computer Engineering (CISCE), Haikou, China, 2019, pp. 411-414, doi: 10.1109/CISCE.2019.00097.

[3] K. Pahwa and N. Agarwal, "Stock Market Analysis using Supervised Machine Learning," 2019 International Conference on Machine Learning, Big Data, Cloud and Parallel Computing (COMITCon), Faridabad, India, 2019, pp. 197-200, doi: 10.1109/COMITCon.2019.8862225.

[4] K. A. Taher, B. Mohammed Yasin Jisan and M. M. Rahman, "Network Intrusion Detection using Supervised Machine Learning Technique with Feature Selection," 2019 International Conference on Robotics,Electrical and Signal Processing Techniques (ICREST), Dhaka, Bangladesh, 2019, pp. 643-646, doi: 10.1109/ICREST.2019.8644161.

[5] Rahul, S. Adhikari and Monika, "NLP based Machine Learning Approaches for Text Summarization," 2020 Fourth International Conference on Computing Methodologies and Communication (ICCMC), Erode, India, 2020, pp. 535-538, doi: 10.1109/ICCMC48092.2020.ICCMC-00099.

[6] I. C. Freeman, A. J. Haigler, S. E. Schmeelk, L. R. Ellrodt and T. L. Fields, "What are they Researching? Examining Industry-Based Doctoral Dissertation Research through the Lens of Machine Learning," 2018 17th IEEE International Conference on Machine Learning and Applications (ICMLA), Orlando, FL, 2018, pp. 1338-1340, doi: 10.1109/ICMLA.2018.00217.

[7] D. Jiang, F. Li, Y. Yang and S. Yu, "A Tomato Leaf Diseases Classification Method Based on Deep Learning," 2020 Chinese Control And Decision Conference (CCDC),
Hefei, China, 2020, pp. 1446-1450, doi: 10.1109/CCDC49329.2020.9164457.

[8] M. R. Ullah, N. A. Dola, A. Sattar and A. Hasnat, "Plant Diseases Recognition Using Machine Learning," 2019 8th International Conference System Modeling and Advancement in Research Trends (SMART), Moradabad, India, 2019, pp. 67-73, doi: 10.1109/SMART46866.2019.9117284.

[9] M. R. Grossman, D. K. Zak, and E. M. Zelinski, "Mobile apps for caregivers of older adults: Quantitative content analysis." 2018 JMIR mHealth and uHealth, 6(7), e162.

[10] H. Ch. "Research methods in library and information science: A content analysis." 2015 Library \& information science research, 37(1), 36-41.

[11] N. A. Azeez, \& C. Van der Vyver. "Security and privacy issues in e-health cloud-based system: A comprehensive content analysis.” 2019 Egyptian Informatics Journal, 20(2), 97-108.

[12] O. Amale and R. Patil, "'IOT Based Rainfall Monitoring System Using WSN Enabled Architecture"," 2019 3rd International Conference on Computing Methodologies and Communication (ICCMC), Erode, India, 2019, pp. 789-791, doi: 10.1109/ICCMC.2019.8819721.

[13] A. Rehman, A. Khan, M. A. Ali, M. U. Khan, S. U. Khan and L. Ali, "Performance Analysis of PCA, Sparse PCA, Kernel PCA and Incremental PCA Algorithms for Heart Failure Prediction," 2020 International Conference on Electrical, Communication, and Computer Engineering (ICECCE), Istanbul, Turkey, 2020, pp. 1-5, doi: 10.1109/ICECCE49384.2020.9179199.

[14] K. Chen, "Indirect PCA Dimensionality Reduction Based Machine Learning Algorithms for Power System Transient Stability Assessment," 2019 IEEE Innovative Smart Grid Technologies - Asia (ISGT Asia), Chengdu, China, 2019, pp. 4175-4179, doi: 10.1109/ISGTAsia.2019.8881370.

[15] P. Sun, D. Wang, V. C. Mok and L. Shi, "Comparison of Feature Selection Methods and Machine Learning Classifiers for Radiomics Analysis in Glioma Grading," in IEEE Access, vol. 7, pp. 102010-102020, 2019, doi: 10.1109/ACCESS.2019.2928975.

[16] P. Nair and I. Kashyap, "Hybrid Pre-processing Technique for Handling Imbalanced Data and Detecting Outliers for KNN Classifier," 2019 International Conference on Machine Learning, Big Data, Cloud and Parallel Computing (COMITCon), Faridabad, India, 2019, pp. 460464, doi: 10.1109/COMITCon.2019.8862250.

[17] P. Raghavan and N. E. Gayar, "Fraud Detection using Machine Learning and Deep Learning," 2019 International Conference on Computational Intelligence and Knowledge Economy (ICCIKE), Dubai, United Arab 
Emirates, 2019, pp. 334-339, doi: 10.1109/ICCIKE47802.2019.9004231.

[18] A. Juneja and N. N. Das, "Big Data Quality Framework: Pre-Processing Data in Weather Monitoring Application," 2019 International Conference on Machine Learning, Big Data, Cloud and Parallel Computing (COMITCon), Faridabad, India, 2019, pp. 559-563, doi: 10.1109/COMITCon.2019.8862267.

[19] S. Hussein, P. Kandel, C. W. Bolan, M. B. Wallace and U. Bagci, "Lung and Pancreatic Tumor Characterization in the Deep Learning Era: Novel Supervised and Unsupervised Learning Approaches," in IEEE Transactions on Medical Imaging, vol. 38, no. 8, pp. 1777-1787, Aug. 2019, doi: 10.1109/TMI.2019.2894349.

[20] S. Lim and H. Han, "Distinguishing Political Parties From Inaugural Speeches Using Machine Learning," 2020 IEEE Conference on Multimedia Information Processing and Retrieval (MIPR), Shenzhen, Guangdong, China, 2020, pp. 97-100, doi: 10.1109/MIPR49039.2020.00026.

[21] S. Ray, "A Quick Review of Machine Learning Algorithms," 2019 International Conference on Machine Learning, Big Data, Cloud and Parallel Computing (COMITCon), Faridabad, India, 2019, pp. 35-39, doi: 10.1109/COMITCon.2019.8862451.

[22] A. Bhatnagar and S. Srivastava, "A Robust Model for Churn Prediction using Supervised Machine Learning," 2019 IEEE 9th International Conference on Advanced Computing (IACC), Tiruchirappalli, India, 2019, pp. 45-49, doi: 10.1109/IACC48062.2019.8971494.

[23] P. Sonar and K. JayaMalini, "Diabetes Prediction Using Different Machine Learning Approaches," 2019 3rd International Conference on Computing Methodologies and Communication (ICCMC), Erode, India, 2019, pp. 367-371, doi: 10.1109/ICCMC.2019.8819841.

[24] L. M. B. Alonzo, F. B. Chioson, H. S. Co, N. T. Bugtai and R. G. Baldovino, "A Machine Learning Approach for Coconut Sugar Quality Assessment and Prediction," 2018 IEEE 10th International Conference on Humanoid, Nanotechnology, Information Technology, Communication and Control, Environment and Management (HNICEM), Baguio City, Philippines, 2018, pp. 1-4, doi: 10.1109/HNICEM.2018.8666315.

[25] A. Lasisi, M. O. Sadiq, I. Balogun, A. Tunde-Lawal and N. Attoh-Okine, "A Boosted Tree Machine Learning Alternative to Predictive Evaluation of Nondestructive Concrete Compressive Strength," 2019 18th IEEE International Conference On Machine Learning And Applications (ICMLA), Boca Raton, FL, USA, 2019, pp. 321-324, doi: 10.1109/ICMLA.2019.00060.

[26] J. Rasheed, H. B. Dogru and A. Jamil, "Turkish Text Detection System from Videos Using Machine Learning and Deep Learning Techniques," 2020 IEEE Third International
Conference on Data Stream Mining \& Processing (DSMP), Lviv, Ukraine, 2020, pp. 116-120, doi: 10.1109/DSMP47368.2020.9204036.

[27] N. A. J. Sufri, N. A. Rahmad, N. F. Ghazali, N. Shahar and M. A. As'ari, "Vision Based System for Banknote Recognition Using Different Machine Learning and Deep Learning Approach," 2019 IEEE 10th Control and System Graduate Research Colloquium (ICSGRC), Shah Alam, Malaysia, 2019, pp. 5-8, doi: 10.1109/ICSGRC.2019.8837068.

[28] R. Katarya and P. Srinivas, "Predicting Heart Disease at Early Stages using Machine Learning: A Survey," 2020 International Conference on Electronics and Sustainable Communication Systems (ICESC), Coimbatore, India, 2020, pp. 302-305, doi: 10.1109/ICESC48915.2020.9155586.

[29] S. Mojrian et al., "Hybrid Machine Learning Model of Extreme Learning Machine Radial basis function for Breast Cancer Detection and Diagnosis; a Multilayer Fuzzy Expert System," 2020 RIVF International Conference on Computing and Communication Technologies (RIVF), Ho Chi Minh City, Vietnam, 2020, pp. 1-7, doi: 10.1109/RIVF48685.2020.9140744.

[30] P. K. Fahira, Z. P. Rahmadhani, P. Mursanto, A. Wibisono and H. A. Wisesa, "Classical Machine Learning Classification for Javanese Traditional Food Image," 2020 4th International Conference on Informatics and Computational Sciences (ICICoS), Semarang, Indonesia, 2020, pp. 1-5, doi: 10.1109/ICICoS51170.2020.9299039.

[31] N. Reamaroon, M. W. Sjoding, K. Lin, T. J. Iwashyna and K. Najarian, "Accounting for Label Uncertainty in Machine Learning for Detection of Acute Respiratory Distress Syndrome," in IEEE Journal of Biomedical and Health Informatics, vol. 23, no. 1, pp. 407415, Jan. 2019, doi: 10.1109/JBHI.2018.2810820.

[32] G. A. Tahir and C. K. Loo, "An Open-Ended Continual Learning for Food Recognition Using Class Incremental Extreme Learning Machines," in IEEE Access, vol. 8, pp. 82328-82346, 2020, doi: 10.1109/ACCESS.2020.2991810.

[33] R. Singh, J. Singh, M. S. Gill, R. Malhotra and Garima, "Transfer Learning Code Vectorizer based Machine Learning Models for Software Defect Prediction," 2020 International Conference on Computational Performance Evaluation (ComPE), Shillong, India, 2020, pp. 497-502, doi: 10.1109/ComPE49325.2020.9200076.

[34] K. A. Taher, B. Mohammed Yasin Jisan and M. M. Rahman, "Network Intrusion Detection using Supervised Machine Learning Technique with Feature Selection," 2019 International Conference on Robotics,Electrical and Signal Processing Techniques (ICREST), Dhaka, Bangladesh, 2019, pp. 643-646, doi: 10.1109/ICREST.2019.8644161. 
[35] K. N. Bhanu, H. J. Jasmine and H. S. Mahadevaswamy, "Machine learning Implementation in IoT based Intelligent System for Agriculture," 2020 International Conference for Emerging Technology (INCET), Belgaum, India, 2020, pp. 1-5, doi: 10.1109/INCET49848.2020.9153978.

[36] T. Toivonen, I. Jormanainen, J. Kahila, M. Tedre, T. Valtonen and $\mathrm{H}$. Vartiainen, "Co-Designing Machine Learning Apps in K-12 With Primary School Children," 2020 IEEE 20th International Conference on Advanced Learning Technologies (ICALT), Tartu, Estonia, 2020, pp. 308-310, doi: 10.1109/ICALT49669.2020.00099.

[37] T. Mehejabin, F. Rahman, S. Yeasmin and M. Sarkar, "Identification of Most Relevant Breast Cancer miRNA using Machine Learning Algorithms," 2020 11th International Conference on Computing, Communication and Networking Technologies (ICCCNT), Kharagpur, India, 2020, pp. 1-6, doi: 10.1109/ICCCNT49239.2020.9225624.

[38] K. Indira, E. Brumancia, P. S. Kumar and S. P. T. Reddy, "Location prediction on Twitter using machine learning Techniques," 2019 3rd International Conference on Trends in Electronics and Informatics (ICOEI), Tirunelveli, India, 2019, pp. 700-703, doi: 10.1109/ICOEI.2019.8862768.

[39] R. Farhat, Y. Mourali, M. Jemni and H. Ezzedine, "An overview of Machine Learning Technologies and their use in E-learning," 2020 International Multi-Conference on: "Organization of Knowledge and Advanced Technologies" (OCTA), Tunis, Tunisia, 2020, pp. 1-4, doi: 10.1109/OCTA49274.2020.9151758.

[40] M. M. Baig et al., "Machine Learning-based Risk of Hospital Readmissions: Predicting Acute Readmissions within 30 Days of Discharge," 2019 41st Annual International Conference of the IEEE Engineering in Medicine and Biology Society (EMBC), Berlin, Germany, 2019, pp. 2178-2181, doi: 10.1109/EMBC.2019.8856646.

[41] S. Satpathy, S. P. Mishra and A. K. Nayak, "Analysis of Learning Approaches for Machine Translation Systems," 2019 International Conference on Applied Machine Learning (ICAML), Bhubaneswar, India, 2019, pp. 160-164, doi: 10.1109/ICAML48257.2019.00038.

[42] R. Tao et al., "Magnetocardiography-Based Ischemic Heart Disease Detection and Localization Using Machine Learning Methods," in IEEE Transactions on Biomedical Engineering, vol. 66, no. 6, pp. 1658-1667, June 2019, doi: 10.1109/TBME.2018.2877649.

[43] R. Trinchero and F. G. Canavero, "Machine Learning for the Design of a Distribution Network for HighSpeed Signals," 2019 International Conference on Electromagnetics in Advanced Applications (ICEAA), Granada, Spain, 2019, pp. 1038-1041, doi: 10.1109/ICEAA.2019.8879415.
[44] A. Halimaa A. and K. Sundarakantham, "Machine Learning Based Intrusion Detection System," 2019 3rd International Conference on Trends in Electronics and Informatics (ICOEI), Tirunelveli, India, 2019, pp. 916-920, doi: 10.1109/ICOEI.2019.8862784.

[45] H. M. E. Misilmani and T. Naous, "Machine Learning in Antenna Design: An Overview on Machine Learning Concept and Algorithms," 2019 International Conference on High Performance Computing \& Simulation (HPCS), Dublin, Ireland, 2019, pp. 600-607, doi: 10.1109/HPCS48598.2019.9188224.

[46] K. Al-Gumaei, A. Müller, J. N. Weskamp, C. S. Longo, F. Pethig and S. Windmann, "Scalable Analytics Platform for Machine Learning in Smart Production Systems," 2019 24th IEEE International Conference on Emerging Technologies and Factory Automation (ETFA), Zaragoza, Spain, 2019, pp. 1155-1162, doi: 10.1109/ETFA.2019.8869075.

[47] E. A. Bayrak, P. Kırc1 and T. Ensari, "Comparison of Machine Learning Methods for Breast Cancer Diagnosis," 2019 Scientific Meeting on Electrical-Electronics \& Biomedical Engineering and Computer Science (EBBT), Istanbul, Turkey, 2019, pp. 1-3, doi: 10.1109/EBBT.2019.8741990.

[48] A. Mujib and T. Djatna, "Ensemble Learning for Predictive Maintenance on Wafer Stick Machine Using IoT Sensor Data," 2020 International Conference on Computer Science and Its Application in Agriculture (ICOSICA), Bogor, Indonesia, 2020, pp. 1-5, doi: 10.1109/ICOSICA49951.2020.9243180.

[49] S. Mirzaei, T. Sidi, C. Keasar and S. Crivelli, "Purely Structural Protein Scoring Functions Using Support Vector Machine and Ensemble Learning," in IEEE/ACM Transactions on Computational Biology and Bioinformatics, vol. 16, no. 5, pp. 1515-1523, 1 Sept.-Oct. 2019, doi: 10.1109/TCBB.2016.2602269..

[50] T. Badriyah, N. Sakinah, I. Syarif and D. R. Syarif, "Machine Learning Algorithm for Stroke Disease Classification," 2020 International Conference on Electrical, Communication, and Computer Engineering (ICECCE), Istanbul, Turkey, 2020, pp. 1-5, doi: 10.1109/ICECCE49384.2020.9179307.

[51] P. S. Gouripeddi, R. Gouripeddi and S. P. Gouripeddi, "Toward Machine Learning and Big Data Approaches for Learning Analytics," 2019 IEEE Tenth International Conference on Technology for Education (T4E), Goa, India, 2019, pp. 256-257, doi: 10.1109/T4E.2019.000-6.

[52] G. Meena, D. Sharma and M. Mahrishi, "Traffic Prediction for Intelligent Transportation System using Machine Learning," 2020 3rd International Conference on Emerging Technologies in Computer Engineering: Machine 
Learning and Internet of Things (ICETCE), Jaipur, India, 2020, pp. 145-148, doi: 10.1109/ICETCE48199.2020.9091758.

[53] P. Chopade, D. Edwards, S. M. Khan, A. Andrade and S. Pu, "CPSX: Using AI-Machine Learning for Mapping Human-Human Interaction and Measurement of CPS Teamwork Skills," 2019 IEEE International Symposium on Technologies for Homeland Security (HST), Woburn, MA, USA, 2019, pp. 1-6, doi: 10.1109/HST47167.2019.9032906. [54] M. R. Ahmed, Y. Zhang, Z. Feng, B. Lo, O. T. Inan and H. Liao, "Neuroimaging and Machine Learning for Dementia Diagnosis: Recent Advancements and Future Prospects," in IEEE Reviews in Biomedical Engineering, vol. 12, pp. 19-33, 2019, doi: 10.1109/RBME.2018.2886237.

[55] J. Song et al., "Demagnetization Modeling Research for Permanent Magnet in PMSLM Using Extreme Learning Machine," 2019 IEEE International Electric Machines \& Drives Conference (IEMDC), San Diego, CA, USA, 2019, pp. 1757-1761, doi: 10.1109/IEMDC.2019.8785136.

[56] I. L. Afonin, A. V. Gorelik, S. S. Muratchaev, A. S. Volkov and E. K. Morozov, "Development of an adaptive TCP algorithm based on machine learning in telecommunication networks," 2019 Systems of Signal Synchronization, Generating and Processing in Telecommunications (SYNCHROINFO), Russia, 2019, pp. 1-5, doi: 10.1109/SYNCHROINFO.2019.8814023.

[57] A. Srivastava, S. Saini and D. Gupta, "Comparison of Various Machine Learning Techniques and Its Uses in Different Fields," 2019 3rd International conference on Electronics, Communication and Aerospace Technology (ICECA), Coimbatore, India, 2019, pp. 81-86, doi: 10.1109/ICECA.2019.8822068.

[58] Kunal and M. Dua, "Machine Learning Approach to IDS: A Comprehensive Review," 2019 3rd International conference on Electronics, Communication and Aerospace Technology (ICECA), Coimbatore, India, 2019, pp. 117-121, doi: 10.1109/ICECA.2019.8822120.

[59] K. Georgiadis, G. van Lankveld, K. Bahreini and W. Westera, "Learning Analytics Should Analyse the Learning: Proposing a Generic Stealth Assessment Tool," 2019 IEEE Conference on Games (CoG), London, UK, 2019, pp. 1-8, doi: 10.1109/CIG.2019.8847960.

[60] P. P. Graybill and M. Kiani, "Eyelid Movement Command Classification Using Machine Learning," 2019 41st Annual International Conference of the IEEE Engineering in Medicine and Biology Society (EMBC), Berlin, Germany, 2019, pp. 3637-3640, doi: 10.1109/EMBC.2019.8857766.

[61] J. Wu, Y. Peng, M. Song, M. Cui and L. Zhang, "Link Congestion Prediction using Machine Learning for Software-Defined-Network Data Plane," 2019 International Conference on Computer, Information and
Telecommunication Systems (CITS), Beijing, China, 2019, pp. 1-5, doi: 10.1109/CITS.2019.8862098.

[62] A. Asokan and J. Anitha, "Machine Learning based Image Processing Techniques for Satellite Image Analysis A Survey," 2019 International Conference on Machine Learning, Big Data, Cloud and Parallel Computing (COMITCon), Faridabad, India, 2019, pp. 119-124, doi: 10.1109/COMITCon.2019.8862452.

[63] R. Vishwakarma and A. K. Jain, "A Honeypot with Machine Learning based Detection Framework for defending IoT based Botnet DDoS Attacks," 2019 3rd International Conference on Trends in Electronics and Informatics (ICOEI), Tirunelveli, India, 2019, pp. 1019-1024, doi: 10.1109/ICOEI.2019.8862720.

[64] M. Tian, H. Chen and G. Liu, "Cloud Detection and Classification for S-NPP FSR CRIS Data Using Supervised Machine Learning," IGARSS 2019 - 2019 IEEE International Geoscience and Remote Sensing Symposium, Yokohama, Japan, 2019, pp. 9827-9830, doi: 10.1109/IGARSS.2019.8898876.

[65] E. Santi et al., "On The Use of Machine Learning and Polarimetry For Estimating Soil Moisture From Radarsat Imagery Over Italian And Canadian Test Sites," IGARSS 2019 - 2019 IEEE International Geoscience and Remote Sensing Symposium, Yokohama, Japan, 2019, pp. 57605763, doi: 10.1109/IGARSS.2019.8900269.

[66] D. Katsaros, G. Stavropoulos and D. Papakostas, "Which machine learning paradigm for fake news detection?" 2019 IEEE/WIC/ACM International Conference on Web Intelligence (WI), Thessaloniki, Greece, 2019, pp. 383-387. [67] S. Nakagawa, T. Hochin, H. Nomiya, H. Nakanishi and M. Shoji, "Prediction of Abnormal Plasma Discharge through Machine Learning," 2019 6th International Conference on Computational Science/Intelligence and Applied Informatics (CSII), Honolulu, HI, USA, 2019, pp. 712, doi: 10.1109/CSII.2019.00009.

[68] T. Goswami and U. B. Roy, "Predictive Model for Classification of Power System Faults using Machine Learning," TENCON 2019 - 2019 IEEE Region 10 Conference (TENCON), Kochi, India, 2019, pp. 1881-1885, doi: 10.1109/TENCON.2019.8929264.

[69] P. Liu, W. Zhang, J. Qiu, Q. Hu and K. He, "Reagent Color Recognition Model for Android Platform Based on OPENCV and Machine Learning," 2019 International Conference on Machine Learning, Big Data and Business Intelligence (MLBDBI), Taiyuan, China, 2019, pp. 270-273, doi: 10.1109/MLBDBI48998.2019.00060.

[70] Y. Yuan, J. Huang and K. Yan, "Virtual Reality Therapy and Machine Learning Techniques in Drug Addiction Treatment," 2019 10th International Conference on Information Technology in Medicine and Education 
(ITME), Qingdao, China, 2019, pp. 241-245, doi: 10.1109/ITME.2019.00062.

[71] S. Ryan, R. Corizzo, I. Kiringa and N. Japkowicz, "Deep Learning Versus Conventional Learning in Data Streams with Concept Drifts," 2019 18th IEEE International Conference On Machine Learning And Applications (ICMLA), Boca Raton, FL, USA, 2019, pp. 1306-1313, doi: 10.1109/ICMLA.2019.00213.

[72] E. Eziama, S. Ahmed, S. Ahmed, F. Awin and K. Tepe, "Detection of Adversary Nodes in Machine-ToMachine Communication Using Machine Learning Based Trust Model," 2019 IEEE International Symposium on Signal Processing and Information Technology (ISSPIT), Ajman, United Arab Emirates, 2019, pp. 1-6, doi: 10.1109/ISSPIT47144.2019.9001743.

[73] H. Lahmer, A. E. Oueslati and Z. Lachiri, "DNA Microarray Analysis Using Machine Learning to Recognize Cell Cycle Regulated Genes," 2019 International Conference on Control, Automation and Diagnosis (ICCAD), Grenoble, France, 2019, pp. 1-5, doi: 10.1109/ICCAD46983.2019.9037868.

[74] N. Lisin and S. Zapechnikov, "Order-preserving Encryption as a Tool for Privacy-Preserving Machine Learning," 2020 IEEE Conference of Russian Young Researchers in Electrical and Electronic Engineering (EIConRus), St. Petersburg and Moscow, Russia, 2020, pp. 2090-2092, doi: 10.1109/EIConRus49466.2020.9039294.

[75] S. Khatri, A. Arora and A. P. Agrawal, "Supervised Machine Learning Algorithms for Credit Card Fraud Detection: A Comparison," 2020 10th International Conference on Cloud Computing, Data Science \& Engineering (Confluence), Noida, India, 2020, pp. 680-683, doi: 10.1109/Confluence47617.2020.9057851.

[76] M. I. Patel, S. Suthar and J. Thakar, "Survey on Image Compression using Machine Learning and Deep Learning," 2019 International Conference on Intelligent Computing and Control Systems (ICCS), Madurai, India, 2019, pp. 1103-1105, doi: 10.1109/ICCS45141.2019.9065473.

[77] U. P. Singh, K. Rakesh, Rishabh, V. Kumar and K. P. Singh, "Improved Coupled Autoencoder based Zero Shot Recognition using Active Learning," 2019 IEEE Conference on Information and Communication Technology, Allahabad, India, 2019, pp. 1-6, doi: 10.1109/CICT48419.2019.9066127.

[78] A. Norouzi, D. Gordon, M. Aliramezani and C. R. Koch, "Machine Learning-based Diesel Engine-Out NOx Reduction Using a plug-in PD-type Iterative Learning Control," 2020 IEEE Conference on Control Technology and Applications (CCTA), Montreal, QC, Canada, 2020, pp. 450455, doi: 10.1109/CCTA41146.2020.9206277.
[79] D. Radovanović and S. Đukanovic, "Image-Based Plant Disease Detection: A Comparison of Deep Learning and Classical Machine Learning Algorithms," 2020 24th International Conference on Information Technology (IT), Zabljak, Montenegro, 2020, pp. 1-4, doi: 10.1109/IT48810.2020.9070664.

[80] K. Chauhan et al., "Automated Machine Learning: The New Wave of Machine Learning," 2020 2nd International Conference on Innovative Mechanisms for Industry Applications (ICIMIA), Bangalore, India, 2020, pp. 205-212, doi: 10.1109/ICIMIA48430.2020.9074859.

[81] S. C. Lee and T. H. Han, "A 4-way Matrix Multiply Unit for High Throughput Machine Learning Accelerator," 2019 International SoC Design Conference (ISOCC), Jeju, Korea (South), 2019, pp. 113-114, doi: 10.1109/ISOCC47750.2019.9078493.

[82] T. Karimi, B. Majidi and M. T. Manzuri, "Deep ESchool-Nurse for Personalized Health-Centered E-Learning Administration," 2019 13th Iranian and 7th National Conference on e-Learning and e-Teaching (ICeLeT), Tehran, Iran, 2019, pp. 1-6, doi: 10.1109/ICELET46946.2019.9091668.

[83] M. A. Bhatti, R. Riaz, S. S. Rizvi, S. Shokat, F. Riaz and S. J. Kwon, "Outlier detection in indoor localization and Internet of Things (IoT) using machine learning," in Journal of Communications and Networks, vol. 22, no. 3, pp. 236243, June 2020, doi: 10.1109/JCN.2020.000018.

[84] S. Karmakar, S. Subhasmita Swain, G. Firdous, S. Mohanty and T. Kumar Mohapatra, "Machine Learning Approach to Estimation of Internal Parameters of a Single Phase Transformer," 2020 International Conference for Emerging Technology (INCET), Belgaum, India, 2020, pp. 1-4, doi: 10.1109/INCET49848.2020.9154161.

[85] R. Talbi, "Towards Practical Privacy-Preserving Collaborative Machine Learning at a Scale," 2020 50th Annual IEEE-IFIP International Conference on Dependable Systems and Networks-Supplemental Volume (DSN-S), Valencia, Spain, 2020, pp. 69-70, doi: 10.1109/DSNS50200.2020.00037.

[86] T. Zoppi, A. Ceccarelli and A. Bondavalli, "Into the Unknown: Unsupervised Machine Learning Algorithms for Anomaly-Based Intrusion Detection," 2020 50th Annual IEEE-IFIP International Conference on Dependable Systems and Networks-Supplemental Volume (DSN-S), Valencia, Spain, 2020, pp. 81-81, doi: 10.1109/DSNS50200.2020.00044.

[87] Y. Ma, K. Zhao, Q. Wang and Y. Tian, "Incremental Cost-Sensitive Support Vector Machine with LinearExponential Loss," in IEEE Access, vol. 8, pp. 149899149914, 2020, doi: 10.1109/ACCESS.2020.3015954.

[88] R. Sharma, B. Kaushik and N. Gondhi, "Character Recognition using Machine Learning and Deep Learning - A 
Survey," 2020 International Conference on Emerging Smart Computing and Informatics (ESCI), Pune, India, 2020, pp. 341-345, doi: 10.1109/ESCI48226.2020.9167649.

[89] M. Shalini and S. Radhika, "Machine Learning techniques for Prediction from various Breast Cancer Datasets," 2020 Sixth International Conference on Bio Signals, Images, and Instrumentation (ICBSII), Chennai, India, 2020, pp. 1-5, doi: 10.1109/ICBSII49132.2020.9167657.

[90] R. K. Devi and G. Elizabeth Rani, "A Comparative Study on Handwritten Digit Recognizer using Machine Learning Technique," 2019 IEEE International Conference on Clean Energy and Energy Efficient Electronics Circuit for Sustainable Development (INCCES), Krishnankoil, India, 2019, pp. 1-5, doi: 10.1109/INCCES47820.2019.9167748.

[91] H. Soni, P. Arora and D. Rajeswari, "Malicious Application Detection in Android using Machine Learning," 2020 International Conference on Communication and Signal Processing (ICCSP), Chennai, India, 2020, pp. 0846-0848, doi: 10.1109/ICCSP48568.2020.9182170.

[92] S. Akshay and T. K. Ramesh, "Efficient Machine Learning Algorithm for Smart Irrigation," 2020 International Conference on Communication and Signal Processing (ICCSP), Chennai, India, 2020, pp. 867-870, doi: 10.1109/ICCSP48568.2020.9182215.

[93] F. O. Olowononi, D. B. Rawat and C. Liu, "Resilient Machine Learning for Networked Cyber Physical Systems: A Survey for Machine Learning Security to Securing Machine Learning for CPS," in IEEE Communications Surveys \& Tutorials, vol. 23, no. 1, pp. 524-552, Firstquarter 2021, doi: 10.1109/COMST.2020.3036778.

[94] B. Sharma, J. K. P. S. Yadav and S. Yadav, "Predict Crop Production in India Using Machine Learning Technique: A Survey," 2020 8th International Conference on Reliability, Infocom Technologies and Optimization (Trends and Future Directions) (ICRITO), Noida, India, 2020, pp. 993-997, doi: 10.1109/ICRITO48877.2020.9197953.

[95] W. Zhe, C. Wei and L. Chunlin, "DoS attack detection model of smart grid based on machine learning method," 2020 IEEE International Conference on Power, Intelligent Computing and Systems (ICPICS), Shenyang, China, 2020, pp. 735-738, doi: 10.1109/ICPICS50287.2020.9202401.

[96] Y. Chang, X. Cui, G. Hou and Y. Jin, "Calibration of the Pressure Sensor Device with the Extreme Learning Machine," 2020 21st International Conference on Electronic Packaging Technology (ICEPT), Guangzhou, China, 2020, pp. 1-5, doi: 10.1109/ICEPT50128.2020.9202675.

[97] H. Shahriar, K. Qian and H. Zhang, "Learning Environment Containerization of Machine Leaning for Cybersecurity," 2020 IEEE 44th Annual Computers, Software, and Applications Conference (COMPSAC),
Madrid, Spain, 2020, pp. 1131-1132, doi: 10.1109/COMPSAC48688.2020.0-105.

[98] Z. Huang, F. Liu, M. Tang, J. Qiu and Y. Peng, "A distributed computing framework based on lightweight variance reduction method to accelerate machine learning training on blockchain," in China Communications, vol. 17, no. 9, pp. 77-89, Sept. 2020, doi: 10.23919/JCC.2020.09.007. [99] M. Kalimuthu, P. Vaishnavi and M. Kishore, "Crop Prediction using Machine Learning," 2020 Third International Conference on Smart Systems and Inventive Technology (ICSSIT), Tirunelveli, India, 2020, pp. 926-932, doi: 10.1109/ICSSIT48917.2020.9214190.

[100] A. Ç. Seçkin, "Adaptive Positioning System Design Using AR Markers and Machine Learning for Mobile Robot," 2020 5th International Conference on Computer Science and Engineering (UBMK), Diyarbakir, Turkey, 2020, pp. 160-164, doi: 10.1109/UBMK50275.2020.9219475.

[101] S. Wibirama, A. P. Sidhawara, G. Lukhayu Pritalia and T. B. Adji, "A Survey of Learning Style Detection Method using Eye-Tracking and Machine Learning in Multimedia Learning," 2020 International Symposium on Community-centric Systems (CcS), Tokyo, Japan, 2020, pp. 1-6, doi: 10.1109/CcS49175.2020.9231447.

[102] S. Banerji, A. Hamrick, A. Majumder, R. Menon and B. Sensale-Rodriguez, "Ultra-compact Design of Power Splitters via Machine Learning," 2020 IEEE Photonics Conference (IPC), Vancouver, BC, Canada, 2020, pp. 1-2, doi: 10.1109/IPC47351.2020.9252230.

[103] G. Tsang, X. Xie and S. -M. Zhou, "Harnessing the Power of Machine Learning in Dementia Informatics Research: Issues, Opportunities, and Challenges," in IEEE Reviews in Biomedical Engineering, vol. 13, pp. 113-129, 2020, doi: 10.1109/RBME.2019.2904488.

[104] M. Roper, "Using Machine Learning to Classify Test Outcomes," 2019 IEEE International Conference On Artificial Intelligence Testing (AITest), Newark, CA, USA, 2019, pp. 99-100, doi: 10.1109/AITest.2019.00009.

[105] M. Zhang and D. Wang, "Machine Learning Based Alarm Analysis and Failure Forecast in Optical Networks," 2019 24th OptoElectronics and Communications Conference (OECC) and 2019 International Conference on Photonics in Switching and Computing (PSC), Fukuoka, Japan, 2019, pp. 1-3, doi: 10.23919/PS.2019.8817991.

[106] W. Caesarendra, T. Wijaya, B. K. Pappachan and T. Tjahjowidodo, "Adaptation to Industry 4.0 Using Machine Learning and Cloud Computing to Improve the Conventional Method of Deburring in Aerospace Manufacturing Industry," 2019 12th International Conference on Information \& Communication Technology and System (ICTS), Surabaya, Indonesia, 2019, pp. 120-124, doi: 10.1109/ICTS.2019.8850990. 
[107] S. Harish and K. S. Gayathri, "Smart Home-based Prediction of Symptoms of Alzheimer's Disease using Machine Learning and Contextual Approach," 2019 International Conference on Computational Intelligence in Data Science (ICCIDS), Chennai, India, 2019, pp. 1-6, doi: 10.1109/ICCIDS.2019.8862163.

[108] A. Yahyaoui, A. Jamil, J. Rasheed and M. Yesiltepe, "A Decision Support System for Diabetes Prediction Using Machine Learning and Deep Learning Techniques," 2019 1st International Informatics and Software Engineering Conference (UBMYK), Ankara, Turkey, 2019, pp. 1-4, doi: 10.1109/UBMYK48245.2019.8965556.

[109] R. Karwa and V. Honmane, "Building Search Engine Using Machine Learning Technique," 2019 International Conference on Intelligent Computing and Control Systems (ICCS), Madurai, India, 2019, pp. 10611064, doi: 10.1109/ICCS45141.2019.9065846.

[110] A. U. HAQ et al., "Identifying the Predictive Capability of Machine Learning Classifiers for Designing Heart Disease Detection System," 2019 16th International Computer Conference on Wavelet Active Media Technology and Information Processing, Chengdu, China, 2019, pp. 130138, doi: 10.1109/ICCWAMTIP47768.2019.9067519.

[111] H. Ouanan and E. H. Abdelwahed, "Image processing and machine learning applications in mining industry: Mine 4.0," 2019 International Conference on Intelligent Systems and Advanced Computing Sciences (ISACS), Taza, Morocco, 2019, pp. 1-5, doi: 10.1109/ISACS48493.2019.9068884.

[112] I. Ortiz Garcés, M. F. Cazares and R. O. Andrade, "Detection of Phishing Attacks with Machine Learning Techniques in Cognitive Security Architecture," 2019 International Conference on Computational Science and Computational Intelligence (CSCI), Las Vegas, NV, USA, 2019, pp. 366-370, doi: 10.1109/CSCI49370.2019.00071.

[113] M. A. Rosales, A. A. Bandala, R. R. Vicerra and E. P. Dadios, "Physiological-Based Smart Stress Detector using Machine Learning Algorithms," 2019 IEEE 11th International Conference on Humanoid, Nanotechnology, Information Technology, Communication and Control, Environment, and Management (HNICEM), Laoag, Philippines, 2019, pp. 1-6, doi: 10.1109/HNICEM48295.2019.9073355.

[114] Q. Wu, Y. Cao, H. Wang and W. Hong, "Machinelearning-assisted optimization and its application to antenna designs: Opportunities and challenges," in China Communications, vol. 17, no. 4, pp. 152-164, April 2020, doi: 10.23919/JCC.2020.04.014.

[115] Y. Xu, "Application Research Based on Machine Learning in Network Privacy Security," 2020 International Conference on Computer Information and Big Data
Applications (CIBDA), Guiyang, China, 2020, pp. 237-240, doi: 10.1109/CIBDA50819.2020.00060.

[116] J. Rahul and M. Sora, "Premature Ventricular Contractions Classification using Machine Learning Approach," 2020 International Conference on Smart Electronics and Communication (ICOSEC), Trichy, India, 2020, pp. 367-370, doi: 10.1109/ICOSEC49089.2020.9215290. 